Draft of August 14, 2018

Preprint typeset using $\mathrm{L}_{\mathrm{A}} \mathrm{EX}$ style emulateapj v. 12/16/11

\title{
SUPERNOVA PTF 12GLZ: A POSSIBLE SHOCK BREAKOUT DRIVEN THROUGH AN ASPHERICAL WIND
}

\author{
Maayane T. Soumagnac ${ }^{1}$, Eran O. Ofek ${ }^{1}$, Avishay Gal-yam ${ }^{1}$, Eli Waxmann ${ }^{1}$, Sivan Ginzburg ${ }^{2}$, Nora Linn \\ Strotjohann $^{3}$, Tom A. Barlow ${ }^{4}$, Ehud Behar ${ }^{5}$, Doron Chelouche ${ }^{6}$, Christoffer Fremling ${ }^{7}$, Noam Ganot ${ }^{1}$, Suvi \\ Gezari $^{8}$, Mansi M. Kasliwal ${ }^{9}$, 'Shai Kaspi ${ }^{11}$, Shrinivas R. Kulkarni ${ }^{9}$, Russ R. Laher ${ }^{11}$, Ari Laor ${ }^{5}$, Dan MaOz $^{12}$, \\ Christopher D. Martin ${ }^{4}$, Ehud Nakar ${ }^{12}$, James D. Neill ${ }^{4}$, Hagai Netzer ${ }^{12}$, Peter E. Nugent ${ }^{13,14}$, Dovi \\ Poznanski $^{12}$, Steve Schulze ${ }^{1}$, Ofer Yaron ${ }^{1}$ \\ Draft of August 14, 2018
}

\begin{abstract}
We present visible-light and ultraviolet $(U V)$ observations of the supernova PTF 12glz. The SN was discovered and monitored in near- $U V$ and $\mathrm{R}$ bands as part of a joint GALEX and Palomar Transient Factory campaign. It is among the most energetic Type IIn supernovae observed to date $\left(\approx 10^{51} \mathrm{erg}\right)$. If the radiated energy mainly came from the thermalization of the shock kinetic energy, we show that PTF 12glz was surrounded by $\sim 1 \mathrm{M}_{\odot}$ of circumstellar material (CSM) prior to its explosive death. PTF $12 \mathrm{glz}$ shows a puzzling peculiarity: at early times, while the freely expanding ejecta are presumably masked by the optically thick CSM, the radius of the blackbody that best fits the observations grows at $\approx 8000 \mathrm{~km} \mathrm{~s}^{-1}$. Such a velocity is characteristic of fast moving ejecta rather than optically thick CSM. This phase of radial expansion takes place before any spectroscopic signature of expanding ejecta appears in the spectrum and while both the spectroscopic data and the bolometric luminosity seem to indicate that the CSM is optically thick. We propose a geometrical solution to this puzzle, involving an aspherical structure of the CSM around PTF 12glz. By modeling radiative diffusion through a slab of CSM, we show that an aspherical geometry of the CSM can result in a growing effective radius. This simple model also allows us to recover the decreasing blackbody temperature of PTF $12 \mathrm{glz}$. SLAB-Diffusion, the code we wrote to model the radiative diffusion of photons through a slab of CSM and evaluate the observed radius and temperature, is made available on-line.

Subject headings: keywords
\end{abstract}

\section{INTRODUCTION}

Type IIn supernovae (SNe) are characterized by prominent and narrow-to-intermediate width Balmer emission lines in their spectra (Schlegel 1990, Filippenko 1997. Smith 2014; Gal-Yam 2016). Rather than a signature of the explosion itself, this spectral specificity is presumably the result of the photoionization of a dense, Hydrogen-

\footnotetext{
${ }^{1}$ Department of Particle Physics and Astrophysics, Weizmann Institute of Science, Rehovot 76100, Israel.

${ }^{2}$ Racah Institute of Physics, The Hebrew University, Jerusalem 91904, Israel.

3 Desy Zeuthen, 15738 Zeuthen, Germany.

${ }^{4}$ California Institute of Technology, 1200 East California

Boulevard, MC 278-17, Pasadena, CA 91125, USA.

${ }^{5}$ Physics Department, Technion Israel Institute of Technology, 32000 Haifa, Israel.

${ }^{6}$ Haifa Center for Theoretical Physics and Astrophysics, Faculty of Natural Sciences, University of Haifa, Haifa 3498838, Israel

${ }^{7}$ Department of Astronomy, The Oskar Klein Center, Stockholm University, AlbaNova, 10691 Stockholm, Sweden.

8 Department of Astronomy, University of Maryland, College Park, MD 20742-2421, USA.

9 Cahill Center for Astrophysics, California Institute of Technology, Pasadena, CA, 91125, USA.

10 Spitzer Science Center, California Institute of Technology, Pasadena, CA 91125.

${ }^{11}$ Infrared Processing and Analysis Center, California Institute of Technology, Pasadena, CA 91125, U.S.A.

12 School of Physics and Astronomy, Tel Aviv University, 69978 Tel Aviv, Israel.

13 Lawrence Berkeley National Laboratory, Berkeley, CA, 94720, USA.

14 Department of Astronomy, University of California, Berkeley, CA, 94720-3411, USA.
}

rich, circumstellar medium (CSM) which is ejected from the SN progenitor prior to the explosion.

The Type IIn class is not a well-defined category of objects, as many $\mathrm{SNe}$ show the characteristic narrow Balmer lines in their spectra, sometime during their evolution. These lines are the signature of an external physical phenomenon highly dependent on the surrounding environment, rather than of any intrinsic property of the explosion. Depending on the spatial distribution and physical properties of the CSM, these lines may persist for days ("flash spectroscopy", Gal-Yam et al. 2014, Khazov et al. 2016, Yaron et al. 2017), weeks (e.g., SN 1998s, Li et al. 1998; Fassia et al. 2000, 2001, SN 2005gl, Gal-Yam et al. 2007, SN 2010mc, Ofek et al. 2013a), or years (e.g., SN 1988Z, Danziger \& Kjaer 1991; Stathakis \& Sadler 1991; Turatto et al. 1993; van Dyk et al. 1993, Chugai \& Danziger 1994|Fabian \& Terlevich 1996; Aretxaga et al. 1999; Williams et al. 2002; Schlegel \& Petre 2006; Smith et al. 2017; 2010 jl, Patat et al. 2011; Stoll et al. 2011; Gall et al. 2014; Ofek et al. 2014c).

In the last decades, the physical picture governing SN IIn explosions and the wider family of "interacting" $\mathrm{SNe}$ - SNe whose radiation can be partially or completely accounted for by the ejecta crashing into a dense surrounding medium - has become clearer (see e.g., Chevalier 1982, Chugai \& Danziger 1994, Chugai et al.|2004, Ofek et al. |2010, Chevalier \& Irwin 2011, Ginzburg \& Balberg 2014, Moriya \& Maeda 2014). In recent years, there is growing evidence that, in the majority of cases, the high-density CSM originates from explosive phenomena taking place in the months to years prior to the SN 
explosion. One piece of evidence supporting this conclusion is the direct detection of the so-called precursors (luminous outbursts) in the months to years prior to the SN explosion (e.g., Foley et al. 2007; Pastorello et al. 2007, Fraser et al. 2013, Ofek et al.| 2013a, 2014b, 2016 Elias-Rosa et al. 2016| Thöne et al.|2017). Several theoretical mechanisms have been suggested to explain extreme mass-loss episodes in the final stages of stellar evolution (e.g., Woosley et al. 2007: Quataert \& Shiode 2012 Chevalier 2012 Soker \& Kashi 2016).

While in normal core-collapse $\mathrm{SNe}$, the radiationmediated shock breaks out upon reaching the stellar surface, producing a strong blast in the $U V$ and $\mathrm{X}$ rays (Nakar \& Sari 2010, Rabinak \& Waxman 2011), in the case of SNe IIn the ejecta may crash into the optically thick CSM. The radiation-dominated and radiationmediated shock runs into the CSM surrounding the star and goes on propagating into it as long as $\tau \gtrsim c / v_{s h}$, where $\tau$ is the optical depth from the shock to the edge of the wind, $v_{s h}$ is the shock velocity, and $c$ is the speed of light (e.g., Ofek et al. 2010). When $\tau \sim c / v_{s h}$, (this condition is verified when the timescale for photons to diffuse from the shocked region to the photosphere becomes comparable to the dynamical timescale of the shock), the shock breaks out: photons diffuse ahead of the shock faster than the ejecta and radiation can escape ahead of the shock (Weaver 1976). After the shock breakout, in the presence of massive CSM above the shock, the radiation-dominated shock transforms into a collisionless shock (Katz et al. |2011; Murase et al. 2011, 2014). The collisionless shock slows down the ejecta and converts its kinetic energy into hard X-ray photons (Katz et al. 2011 Murase et al. 2011, 2014). If the optical depth of the CSM above the shock is high enough, the X-rays generated in the collisionless shock are converted into $U V$ and visible radiation (e.g., Chevalier \& Irwin 2012 Svirski et al. 2012). Without a sufficient optical depth though, the bulk of the X-ray photons will not convert into optical photons.

As far as a spectral signature is concerned, the common picture explaining SNe IIn observations is as follows. As long as the CSM is optically thick, the photosphere which emits the continuum is located in the unshocked CSM, masking the observer's view of the shock. The radiation from the shock propagates upstream and photoionizes the slowly moving CSM, resulting in relatively narrow Balmer recombination emission lines in the SN spectrum. As the shock reaches the optically thin medium, broader components can appear in the spectrum - maybe arising from the shocked zone forming at the contact discontinuity between the decelerated ejecta and the shocked CSM (Chugai et al. 2004). Alternatively, if the CSM is optically thin, the lines may be generated in inner regions (e.g., Chevalier \& Fransson 1994)

Observing SNe $1 \mathrm{ln}$ at wavelengths where the collisionless shock radiates most - namely $U V$ and X-rays - has the potential to unveil precious information about the explosion mechanism and the CSM properties (e.g., Ofek et al. 2013b). In particular, it may provide a much better estimate of the bolometric luminosity of the event. In this paper, we present and analyse the $U V$ and visiblelight observations of PTF 12glz, a SN IIn observed in a joint campaign by GALEX and the Palomar Transient Factory (PTF) and detected in the $U V$. PTF 12glz is one of the six SNe discovered during this campaign (Ganot et al. 2016). The survey was carried out as a proof-ofconcept for the ULTRASAT mission (Sagiv et al. 2014).

Observations of SNe IIn are usually analyzed within the framework of spherically symmetric models of CSM. However, resolved images of stars undergoing considerable mass loss (e.g., $\eta$ Carinae; Davidson \& Humphreys 1997, 2012), as well as polarimetry observations (Leonard et al. 2000, Hoffman et al. 2008; Wang \& Wheeler 2008; Reilly et al. 2017) suggest that asphericity should be taken into account for more realistic modeling. Asphericity of the CSM has recently been invoked to interpret the spectrocopic and spectropolarimetric observations of the Type IIn SN SN2012ab (Bilinski et al. 2017). In this paper, we show that the light curve of PTF 12glz may be interpreted as evidence for aspherical CSM.

We present the aforementioned observations of PTF 12glz in $\S 2$. In $\S 3$, we present the analysis of these observations and the puzzling inconsistency between the spectroscopic and photometric observations. In $\S 4$, we model the radiative diffusion of photons through an aspherical slab and propose a solution to this puzzle. We then summarize our main results in $\S 5$. In the Appendix, we make available SLAB-Diffusion, a computer code for modeling radiation through a slab of CSM.

\section{OBSERVATIONS AND DATA REDUCTION}

In this section, we present the observations of PTF $12 \mathrm{glz}$ by the GALEX/PTF $U V$ wide-field transient survey. This campaign, conducted during a nine-week period from 2012 May 24 through 2012 July 28, used the GALEX NUV camera to cover a total area of about 600 $\operatorname{deg}^{2}$ over 20 times with a three-day cadence, while PTF observed the same region with a two-day cadence (Ganot et al. 2016).

\subsection{Discovery}

PTF 12glz was discovered on 2012 July 7 by the PTF (Law et al. 2009: Rau et al. 2009) automatic pipeline reviewing potential transients in the data from the PTF camera mounted on the $1.2 \mathrm{~m}$ Samuel Oschin telescope (P48, Rahmer et al. 2008). The image processing pipeline is discussed in Laher et al. (2014) and the photometric calibration is described in Ofek et al. (2012). The SN is associated with an $r=18.51 \mathrm{mag}$ galaxy, SDSS ${ }^{15}$ J155452.95+033207.5, shown in Figure 1 1 The coordinates of the object, measured in the PTF images are $\alpha=15^{h} 54^{m} 53^{s} .04, \delta=+03^{d} 32^{\prime} 07^{\prime \prime} .5$ (J2000.0). The redshift $z=0.0799$ and the distance modulus $\mu=37.77$ were obtained from the spectrum and the extinction was deduced from Schlafly \& Finkbeiner (2011) and using the extinction curves of Cardelli et al. (1989). All these parameters are summarized in Table 1 .

Previous PTF observations were obtained in the years prior to the SN explosion and the most recent nondetection was on 2012 June 25 . We present a derivation of the explosion epoch in $\S 3.3$.

\subsection{Photometry}

PTF 12glz was observed in multiple bands for almost three years after discovery. The SN was monitored during a rising phase $(t<36$ days $)$ and a decay phase

\footnotetext{
15 Sloan Digital Sky Survey; York et al. (2000)
} 


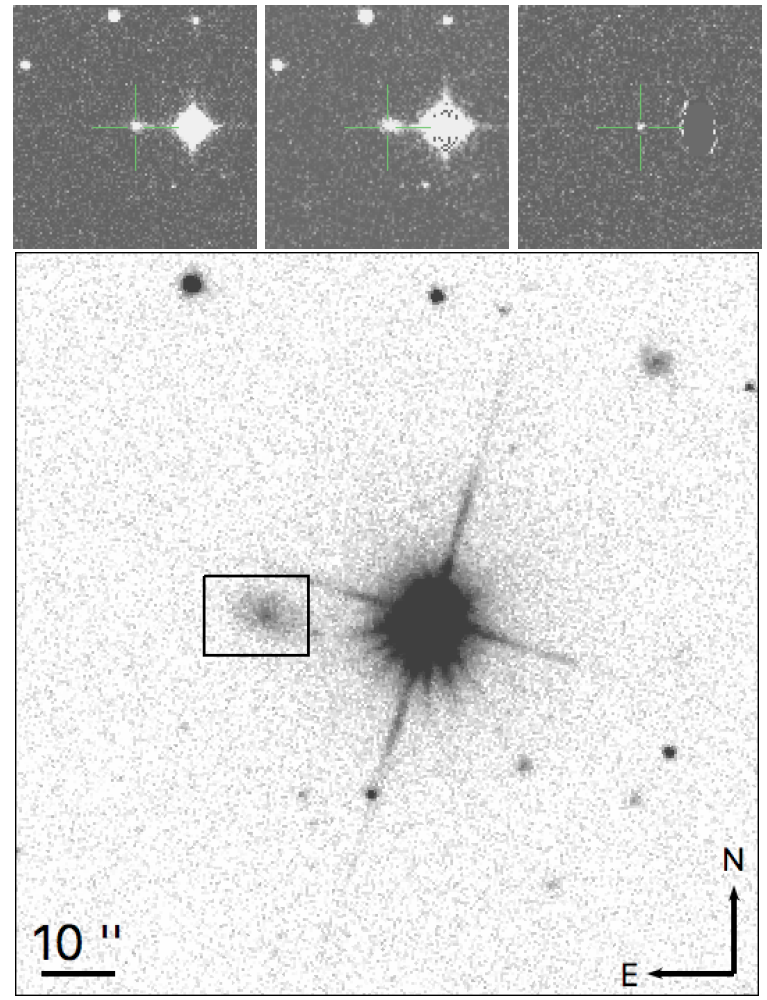

FIG. 1.- Top panels: (left to right) the discovery image, reference image, and subtracted P48 image of PTF 12glz. Lower panel: the SDSS image of J155452.95+033207.5, the host of the supernova PTF 12glz. The box encircles the host: $\alpha=238.72100^{\circ}$ and $\delta=$ $3.53542^{\circ}$. Credit: SDSS.

TABLE 1

\begin{tabular}{lr}
\hline \hline \multicolumn{1}{c}{ Parameter } & \multicolumn{1}{c}{ Value } \\
\hline right ascension $\alpha(J 2000)$ & $238.721000 \mathrm{deg}$ \\
declination $\delta(J 2000)$ & $3.535421 \mathrm{deg}$ \\
redshift $z$ & $z=0.0799$ \\
distance modulus $\mu$ & $37.77 \mathrm{mag}$ \\
galactic extinction $E_{B-V}$ & $0.13 \mathrm{mag}$
\end{tabular}

Note. - Summary of PTF 12glz observational parameters.

$(t>243$ days) but not around peak luminosity. All the light curves are shown in Figure 2. The photometry is reported in electronic Table 2 and is be available via WISeREP 16

GALEX observations of the PTF 12glz field started on 2012 May 26 and 15 observations were obtained with a cadence of $\sim 3$ days. The GALEX NUV camera was operating in scanning mode and observed strips of sky in a drift-scan mode with an effective average integration time of $80 \mathrm{~s}$, to a $N U V$ limiting magnitude of $20.6 \mathrm{mag}$ $[\mathrm{AB}]$. The GALEX data reduction was done using tools ${ }^{17}$ by Ofek (2014).

The P48 telescope was used with a $12 \mathrm{~K} \times 12 \mathrm{~K}$ CCD mosaic camera (Rahmer et al. 2008) and a Mould $R$ -

\footnotetext{
16 https://wiserep.weizmann.ac.il

17 MATLAB Astronomy \& Astrophysics Toolbox, https://webhome.weizmann.ac.il/home/eofek/matlab/
}

TABLE 2

\begin{tabular}{llll}
\hline \hline Epoch (days) & Counts (arb.) & Mag (magAB) & Instrument \\
\hline 11.24 & $0.75 \pm 0.13$ & $20.38 \pm 0.28$ & GALEXnUV \\
9.71 & $362.30 \pm 86.30$ & $20.60 \pm 0.26$ & P48/R \\
185.48 & - & $19.01 \pm 0.06$ & P60/g' \\
185.48 & - & $18.85 \pm 0.04$ & P60/r' \\
185.48 & - & $18.40 \pm 0.05$ & P60/i' \\
\hline
\end{tabular}

Note. - Photometry. This table is available in its entirety in machine-readable format in the online journal. A portion is shown here for guidance regarding its form and content. Time is shown relative to the estimated epoch at which the extrapolated light curve (based on Equation 1 is crossing zero: $t_{0}=2456097.58(2012$ June 19), as derived in $\S 3.3$ To compute the apparent magnitudes from the counts, the zero-point for the $n U V$ data is $Z P_{n U V}=20.08$ and the zero-point for the $\mathrm{P} 48$ data is $Z P_{P 48}=27.00$.

band filter. Data were obtained with a cadence of $\sim 2$ days, to a limiting magnitude of $\mathrm{R} \approx 21 \mathrm{mag}[\mathrm{AB}]$. For the data reduction of the $\mathrm{P} 48$ data, we used a pipeline developed by Mark Sullivan (Sullivan et al. 2006: Firth et al. 2015).

The robotic $1.52 \mathrm{~m}$ telescope at Palomar (P60; Cenko et al. 2006) was used with a $2048 \times 2048$-pixel CCD camera and $g^{\prime}, r^{\prime}, i^{\prime}$ SDSS filters. Data reduction of the P60 data was performed using the FPipe pipeline (Fremling et al. 2016). We calibrated the P60 data in the following way. The $r$-band light curve was scaled so that its average value during the time window covered by both telescopes matches the average value of the P $48 R$-band photometric data. The $g$-band and $i$-band data were scaled to match the synthetic photometry of the calibrated spectroscopic data $(\S 2.3)$. The synthetic photometry used for the calibration and for other purposes in this paper was computed with the PyPhot ${ }^{18}$ pipeline (Fouesneau, in preparation).

Although the photometric data available for PTF $12 \mathrm{glz}$ do not cover the peak, the data during the rise and decay allow to place an upper limit on the absolute magnitude at peak: with $M_{r} \lesssim-20$, PTF $12 \mathrm{glz}$ is at the bright-end of the observed SNe IIn, together with e.g., SN 2006gy (Ofek et al. 2007, Smith \& McCray 2007), SNe 2008fq (Thrasher et al. 2008; Taddia et al. 2013) or SN 2003ma (Rest 2009 Rest et al. 2011). In particular, it is brighter than all SNe in the sample by Kiewe et al. (2012), which was designed to be unbiased.

\subsection{Spectroscopy}

Four optical spectra of PTF 12glz were obtained using the telescopes and spectrographs listed in Table 3 . The two first spectra were taken during the light curve rise, and the two last ones during the decay, at the dates shown in Table 3. The spectra were used to determine the redshift $z=0.0799$ from the narrow host lines $(\mathrm{H} \alpha$ and $[\mathrm{OIII}])$. All the observations were corrected for a galactic extinction of $E_{B-V}=0.13 \mathrm{mag}$, deduced from Schlafly \& Finkbeiner (2011) and using Cardelli et al. (1989) extinction curves.

The spectroscopic observations were calibrated in the following way: the first two and the last spectra, for which we have contemporaneous $\mathrm{P} 48 R$-band data, were scaled so that their synthetic photometry matches the

\footnotetext{
18 http://mfouesneau.github.io/docs/pyphot/
} 


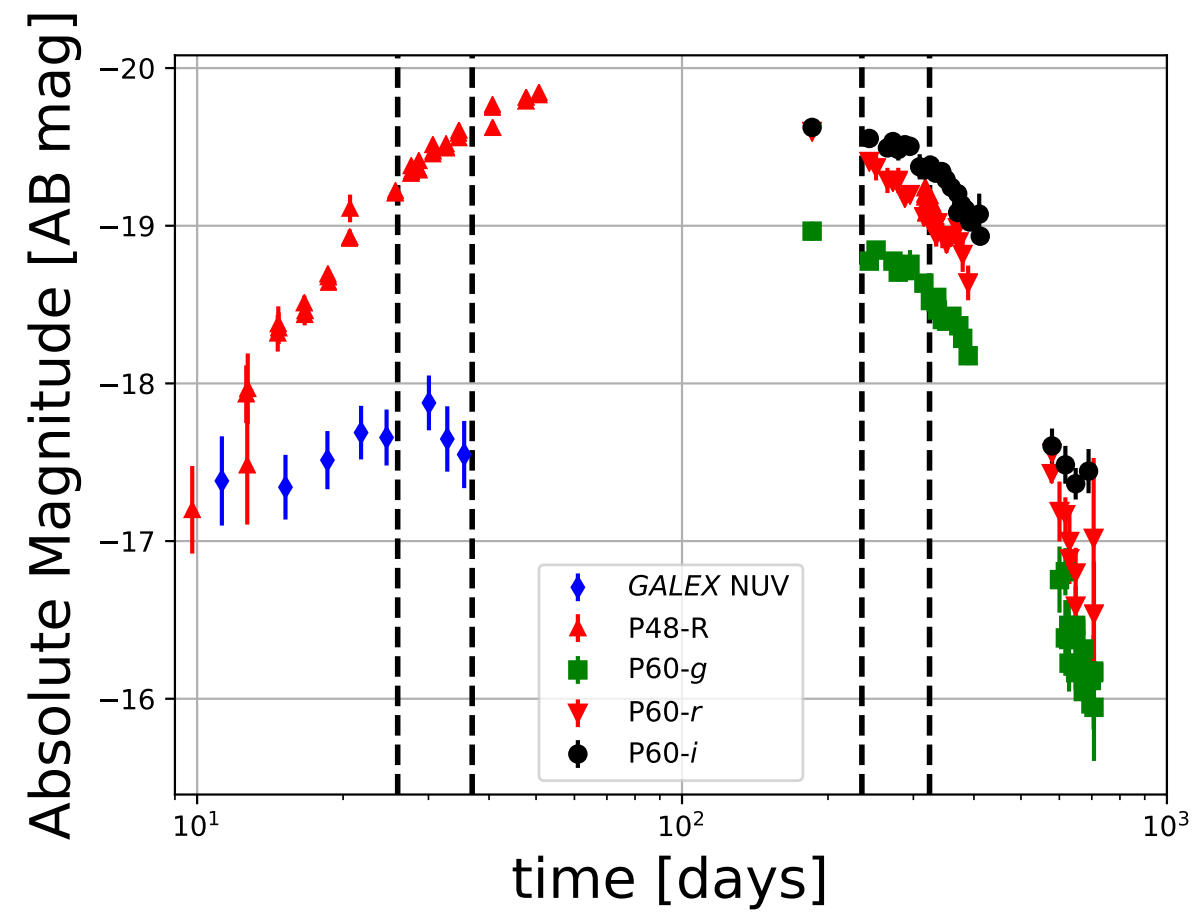

FIG. 2. - The light curve of PTF 12glz. Time is shown relative to the estimated epoch at which the extrapolated light curve (Equation 1 ) is crossing zero: $t_{0}=2456097.58$ (2012 June 19), as derived in $\S 3.3$ Black dashed lines indicate dates at which spectroscopic data exist.

TABLE 3

\begin{tabular}{lll}
\hline \multicolumn{1}{c}{ Date } & Facility & \multicolumn{1}{c}{ Reference } \\
\hline 2012 July 15 & P200 & Oke \& Gunn $(1982)$ \\
2012 July 26 & P200 & - \\
2013 February 9 & LRIS & Oke et al. 1994) \\
2013 May 9 & LRIS & -
\end{tabular}

Note. - Spectroscopic observations of PTF 12glz. The double-beam spectrometer (Oke \& Gunn 1982) mounted on the 200 " Hale telescope at

Palomar was used with a 1" slit, the $5500 \AA$ dichroic and the $316 / 7150$ grating positionned at a grating angle of $24 \mathrm{deg} 38.2 \mathrm{~min}$. The Low-Resolution Imaging Spectrometer (LRIS) (Oke et al. 1994) spectrometer mounted on the $10 \mathrm{~m}$ Keck 1 telescope was used with a 1 " slit, the $5600 \AA$ dichroic and the $400 / 3400$ grism on the blue side.

P48 $R$-band value. The third spectrum was scaled in the same way using the overlapping P60 $r$-band data instead.

The first and last spectra are shown in Figure 3 (the first two spectra are very similar, as well as the last two spectra) and all spectra are available from the Weizmann Interactive Supernova data REPository ${ }^{19}$ (WISeREP, Yaron \& Gal-Yam 2012).

\section{ANALYSIS}

\subsection{Spectroscopy}

The two early spectra, obtained during the rise of the light curve, are characteristic of interacting SNe: a blue continuum with strong and narrow Balmer lines, as well as weak He I (5876, $7065 \AA)$ narrow lines. At short wavelengths, the spectra show absorption from iron, as seen, for example, in SN 2010j 20

We fitted a blackbody spectrum to the six-point spectral energy distribution (corrected for redshift and extinction) obtained by combining (1) the observed photometry in the $N U V$ and $\mathrm{P} 48 R$-band (2) the synthetic photometry of the spectra in the $g, i$ and $r$ SDSS bands. The best-fit temperatures and radii are shown in Table 4 and Figure 6 (as stars). In Figure 3, we show the synthetic and observed photometry derived for the earliest spectrum, on which is superimposed the calibrated spectrum and the best blackbody fit.

Both early spectra show strong and narrow Balmer lines, which for SNe IIn are interpreted as coming from the slow, unshocked, photoionized CSM. Their broad Lorentzian wings may be the signature of electron scattering, as the $\mathrm{H} \alpha$ photons diffuse ahead of the shock through the dense CSM (e.g., Chugai 2001). After subtracting the best-fit continuum from the spectra, we fitted the narrow $\mathrm{H} \alpha$ lines. We tried several linear combinations of Gaussian and Lorentzian functions: the best fit is a superposition of a narrow Gaussian component with $\mathrm{FWHM} \approx 100-200 \mathrm{~km} \mathrm{~s}^{-1}$ (i.e., unresolved), which we interpret as tracing the slow unshocked CSM and an intermediate Lorentzian component with FWHM $\approx$ $500-700 \mathrm{~km} \mathrm{~s}^{-1}$ (if some of the line-broadening comes from electron scattering, this is an upper limit of the CSM speed). The derived speeds and offsets are shown in Table 4. Figure 5 shows the line and the best fit for the latest spectrum.

No signature of expanding material is visible in the

19 https://wiserep.weizmann.ac.il

20 https://wiserep.weizmann.ac.il 

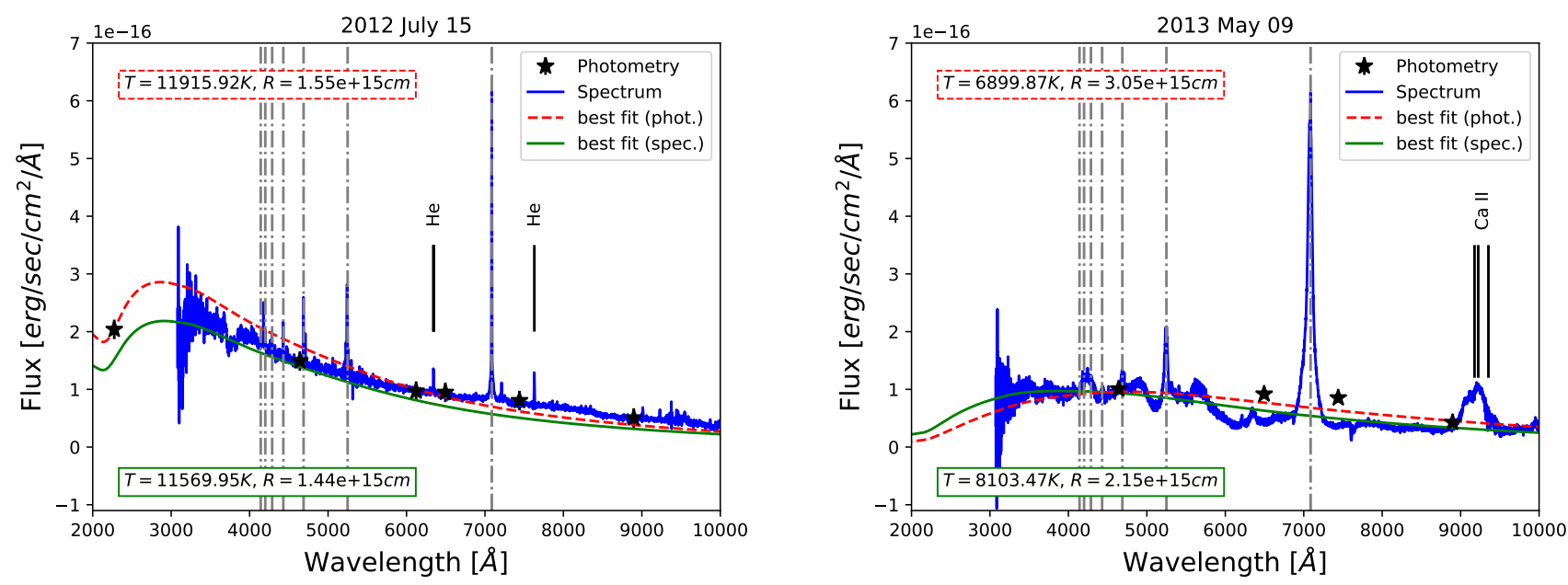

Fig. 3. - The earliest (left) and latest (right) observed spectra of PTF 12glz. Both spectra were calibrated to the $R$-band photometric measurement. Dashed lines indicate the redshifted emission lines for the Balmer series. Black stars show the combination of the observed ( $N U V$ and P48 $R$-band) and synthetic ( $g, r$ and $i$ sdss bands) photometry. The dashed red line shows the blackbody curves that best fits the photometric data (and the best fit values are shown in the box with dashed contours), while the green continuous line shows the blackbody curve that bests fit to the spectroscopic data (and the best fit values are shown in the box with dashed contours). (A color version of this figure is available in the online journal).

spectrum at this stage, which is consistent with a thick CSM obstructing the view of the SN ejecta at early times. We will show in $\S 3.2$ how the photometric data are inconsistent with this picture.

The fits mentioned above, as well as all those mentioned in the rest of this paper, were performed using the emcee algorithm (Foreman-Mackey et al. 2013) to sample from the posterior probability distribution. We then used the ten combinations with the lowest $\chi^{2}$ from the Monte Carlo Markov chain as initial conditions for an optimization algorithm to compute the best fit value (the maximum a posteriori value, in the terminology of Hogg et al. 2010). When errors are noted, they correspond to the $1 \sigma$ limits of the marginalized posterior distributions.

The interpretation of the late-time spectra of Type IIn SNe should be made with the complexity caused by CSM interaction in mind. In "normal" type II SNe, the ejecta that become optically thin at late times are heated from the inside by two sources of energy: (1) remaining thermal deposition from the original heat of the explosion and (2) radioactivity. The late, or "nebular" spectrum shows no clear continuum, and its emission lines reflect the expansion of the ejecta in which they formed. In $\mathrm{SNe}$ IIn, the CSM interaction may continue to dominate the spectrum at late times, e.g., because the ejecta is heated by the shock wave propagating backward from the CSM into its outermost layers (Chevalier \& Fransson 2003).

The two late-time spectra of PTF $12 \mathrm{glz}$ look similar (the latest spectrum is shown in Figure 3): they show a weak continuum in the red, a pseudo-continuum presumably formed by the superposition of narrow [Fe II] emission lines in the blue (e.g. Kiewe et al. 2012) and several broad emission lines. The temperatures and radii derived by fitting a blackbody curve to the observed spectrum are listed in Table 4 and compared to the parameters derived from photometry in Figure 6. Both spectra show broad Balmer emission lines. The derived speeds and redshifts are presented in Table 4 and Fig-

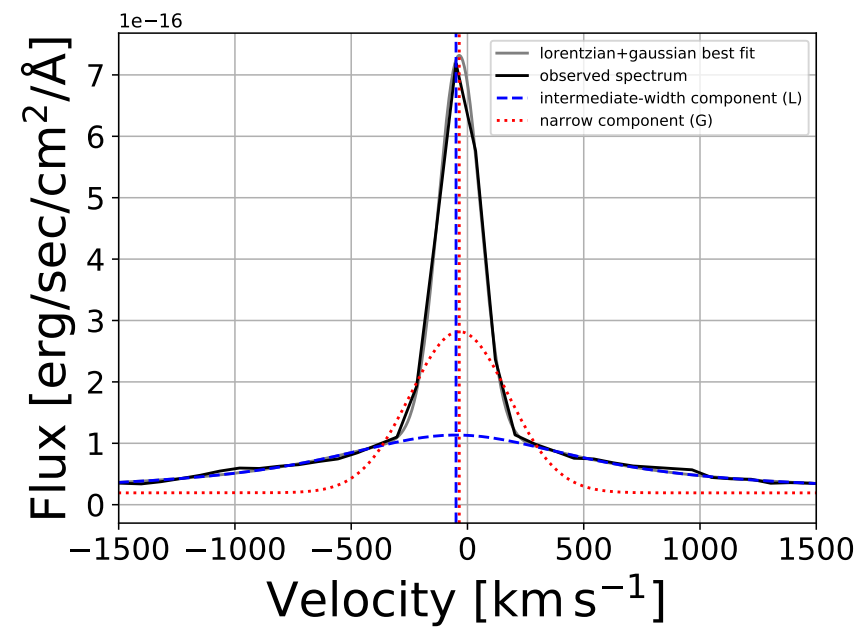

FIG. 4.- The H $\alpha$ line profile on 2012 July 15 (earliest spectrum). The bold black line shows the observed spectrum. The red dotted line and blue dashed line are the narrow Gaussian (G) and intermediate-width Lorentzian (L) whose linear combination (in grey continuous line) best fits the data. The origin of the x-axis indicates the location of the $\mathrm{H} \alpha$ line at the host galaxy, at redshift $z=0.0799$. The vertical lines indicate the center of each component: the narrow component is blueshifted by $\sim-30 \mathrm{~km} \mathrm{~s}^{-1}$ and the intermediate-width component is blueshifted by $\sim-50 \mathrm{~km} \mathrm{~s}^{-1}$.

ure 5 shows the line and the best fit for the latest spectrum. The $\mathrm{H} \alpha$ lines are best fit by a linear combination of an intermediate-width Gaussian component with $F W H M \approx 2000 \mathrm{~km} \mathrm{~s}^{-1}$ that is offset to the blue by $\approx 250 \mathrm{~km} \mathrm{~s}^{-1}$ relative to the galaxy rest-frame and a broad Gaussian component with $F W H M \approx 8000 \mathrm{~km} \mathrm{~s}^{-1}$ that is offset to the blue by $\approx 1000 \mathrm{~km} \mathrm{~s}^{-1}$ relative to the galaxy rest frame. The intermediate-width component could come from the shocked gas in a cold dense shell forming at the contact discontinuity between the decelerated ejecta and the shocked CSM, which is reheated by $\mathrm{X}$-rays and $U V$ radiation from the shock. The broad 


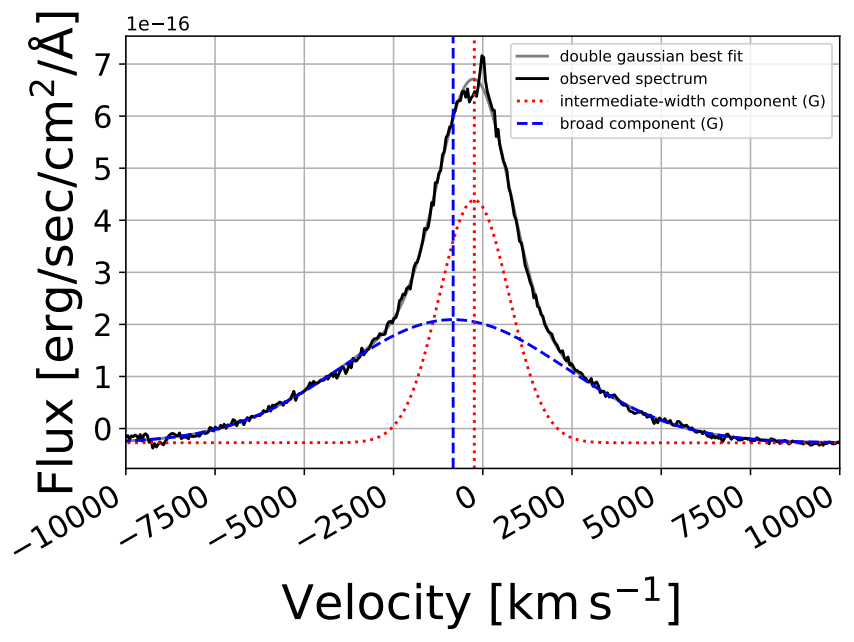

FIG. 5. - The $\mathrm{H} \alpha$ line profile on 2013 May 09 (latest spectrum). The bold black line shows the observed spectrum. The red dotted line and blue dashed line are the intermediate-width and broad Gaussian (G) components whose linear combination (in grey) best fits the data. The vertical lines indicate the center of each component: the narrow component is blueshifted by $\approx$ $-230 \mathrm{~km} \mathrm{~s}^{-1}$ and the intermediate-width component is blueshifted by $\approx-830 \mathrm{~km} \mathrm{~s}^{-1}$.

component velocities are too high to originate from the CSM: they are rather characteristic ejecta velocity values. We deduce from these velocities that the ejecta has - at least partially - emerged through the optically thick layers of the CSM.

At short wavelengths, the spectra show a blue bump that we interpret as a blend of Fe II narrow P-cygni profiles, which may come from the initial metalicity abundance of the star (Kiewe et al. 2012).

Both late spectra also show emission from the the $\mathrm{Ca}$ [II] IR triplet. This is commonly seen in late timeinteracting SNe, e.g., in the type IIn SN 2005ip (Boles et al. 2005; Stritzinger et al. 2012), the type Ic SN 2007dio (Kuncarayakti et al. 2018) and the type Ia-csm PTF 11kx (Dilday et al. 2012; Silverman et al. 2013, Graham et al. 2017).

\subsection{The peculiar evolution of the blackbody radius}

Taking advantage of the multiple-band photometry coverage, we derived the temperature and radius of the blackbody that best fits the photometric data at each epoch (after interpolating the various data sets to obtain data coverage of coinciding epochs, and deriving the errors at the interpolated points with Monte Carlo Markov chain simulations). The derived best-fit temperatures $T_{B B}$ and radii $r_{B B}$ are shown in Figure 6 .

In most epochs, this method implies fitting a blackbody spectrum to a two-point spectral energy distribution. Comparison with results derived from more constraining data detailed in $\S 3.1$ - either spectroscopy (shown in red) or a combination of observed and synthetic photometry (shown in green) - suggests that this method leads to a slight overestimation of the radius and underestimation of the temperature.

The temperature $T_{B B}$ drops from $\approx 15000 \mathrm{~K}$ to $\approx$ $8000 \mathrm{~K}$ during the rise and is stable at $\approx 6000 \mathrm{~K}$ during the decay phase. The decrease of $T_{B B}$ at early time is well fitted by a power law $t^{n}$ with index $n=-0.6$ and is consistent with the temperature evolution observed in the sample by Taddia et al. (2013). However, PTF 12glz is relatively hot compared to the SNe IIn of this sample, where temperatures span between $11500 \mathrm{~K}$ and $5500 \mathrm{~K}$, and compared to other well studied SNe IIn (e.g., 2006gy, Ofek et al. 2007; Smith \& McCray 2007; SN 2005ip, Smith et al. 2009, SN 2010jl, Ofek et al. 2014c).

The derived radius grows by an order of magnitude, from $r_{B B} \approx 6 \times 10^{14} \mathrm{~cm}$ to $r_{B B} \approx 3 \times 10^{15} \mathrm{~cm}$ during the rising phase and is $\approx 4 \times 10^{15} \mathrm{~cm}$ during the light curve decay phase. Such a rise is puzzling within a picture where the optically thick CSM is supposed to mask the shock and the expanding material. To our knowledge, the measured blackbody radius of all SNe IIn observed to date either stalls after a slight increase (e.g., 2005kj, 2006bo, 2008fq, 2006qq, Taddia et al. 2013, 2006tf, Smith et al. 2008), or stays relatively constant at early times (e.g., SN2010jl Ofek et al. 2014c), or even supposedly shrinks (e.g., SN2005ip; SN2006jd, Taddia et al.|2013). Whereas a constant radius is consistent with the continuum photosphere being located in the unshocked optically thick CSM, the possible presence of clumps in the CSM that may expose underlying layers, has been invoked by Smith et al. (2008) to interpret observations of a stalling or shrinking radius.

In our case, the velocity at which $r_{B B}$ grows, reaches $\approx 8000 \mathrm{~km} \mathrm{~s}^{-1}$, a value too large to trace the unshocked layers of the CSM or even the reverse shock. One may naively think that if the CSM was optically thin, then the observed radius would grow. However, in this case, it would be hard to convert efficiently the hard X-ray produced by the colisionless shock into optical radiation. In $\S 5$ we propose one possible geometrical solution to this puzzle.

\subsection{Bolometric light curve}

Based on the measurement of $r_{B B}$ and $T_{B B}$, we were able to derive the luminosity $L_{B B}=4 \pi R^{2} \sigma T^{4}$ of the blackbody fits, shown in Figure 7. Since PTF $12 \mathrm{glz}$ was not observed at peak luminosity, we can only derive a lower limit on the peak luminosity $L_{B B, p e a k}>$ $4 \times 10^{43} \mathrm{erg} \mathrm{s}^{-1}$. This makes PTF $12 \mathrm{glz}$ more luminous than SN 2008fq - the brightest SN of the Taddia et al. (2013) sample - and brighter than all but one SNe of the sample by Ofek et al. (2014a). This suggests that PTF 12glz is at the bright end of the SNe IIn luminosity range.

We fitted the light curve during the rise time with a function of the form

$$
L=L_{\max }\left\{1-\exp \left[\left(t_{0}-t\right) / t_{c}\right]\right\},
$$

(where $t_{0}$ is the time of zero flux, $L_{\max }$ is the maximum bolometric luminosity, and $t_{c}$ is the characteristic rise time of the bolometric light curve). This allowed us to estimate the epoch at which the extrapolated light curve is crossing zero, which is used throughout this paper as the reference time $t_{0}(\mathrm{MJD})=56097.58$.

As shown in Figure 7, $L_{B B}$ decreases about twice as slowly as the $0.98 \mathrm{mag} / 100 \mathrm{~d}$ decline rate characteristic of the radioactive decay of ${ }^{56} \mathrm{Ni}$ to ${ }^{56} \mathrm{Co}$ and then to ${ }^{56} \mathrm{Fe}$. If this decline was produced by the radioactive decay of ${ }^{56} \mathrm{Ni}$, a Nickel mass of at least $14 \mathrm{M}_{\odot}$ would be required to reach the bolometric luminosity of PTF 12glz (see Fig- 
TABLE 4

\begin{tabular}{lllllllll}
\hline \hline Date & \multicolumn{3}{c}{ Continuum } & \multicolumn{2}{c}{ H $\alpha$ narrow comp. } & \multicolumn{2}{l}{ H $\alpha$ intermediate comp. } & \multicolumn{2}{c}{ H $\alpha$ broad comp. } \\
\hline & $T_{B B}$ & $\begin{array}{l}R_{B B} \\
{[\mathrm{~K}]}\end{array}$ & $\begin{array}{l}\Delta v \\
{\left[10^{15} \mathrm{~cm}\right]}\end{array}$ & $\begin{array}{l}F W H M \\
{\left[\mathrm{~km} \mathrm{~s}^{-1}\right]}\end{array}$ & $\begin{array}{l}\Delta v \\
{\left[\mathrm{~km} \mathrm{~s}^{-1}\right]}\end{array}$ & $\begin{array}{l}F W H M \\
{\left[\mathrm{~km} \mathrm{~s}^{-1}\right]}\end{array}\left[\mathrm{km} \mathrm{s}^{-1}\right]$ & $\begin{array}{c}\Delta v \\
{\left[\mathrm{~km} \mathrm{~s}^{-1}\right]}\end{array}$ & $\begin{array}{c}F W H M \\
{\left[\mathrm{~km} \mathrm{~s}^{-1}\right]}\end{array}$ \\
\hline & $13440_{-460}^{+830}$ & $1.31_{-0.14}^{+0.14}$ & -35 & 110 & -50 & 680 & - & - \\
2012 July 15 & $11310_{-460}^{+1080}$ & $1.91_{-0.32}^{+0.22}$ & -80 & 240 & -100 & 530 & - & - \\
2012 July 26 & $8200_{-350}^{+340}$ & $2.56_{-0.20}^{+0.24}$ & - & - & -270 & 1990 & -1070 & 8580 \\
2013 February 09 & $8100_{-390}^{+420}$ & $2.15_{-0.22}^{+0.26}$ & - & - & -240 & 2310 & -830 & 7470 \\
2013 May 09 & & & & & & & &
\end{tabular}

Note. - The table shows the best fit values of the continuum and $\mathrm{H} \alpha$ lines in the four spectra of PTF 12glz. The early spectra are best fit with a linear combination of a narrow Gaussian component (left column) and an intermediate-width Lorentzian component (central column). The late spectra are best fit with a linear combination of an intermediate-width Gaussian component (central column) and a broad Gaussian component (right column). $\Delta z$ is the shift of the centre of each component compared to the galaxy rest frame (the negative values mean that the component is blueshifted).
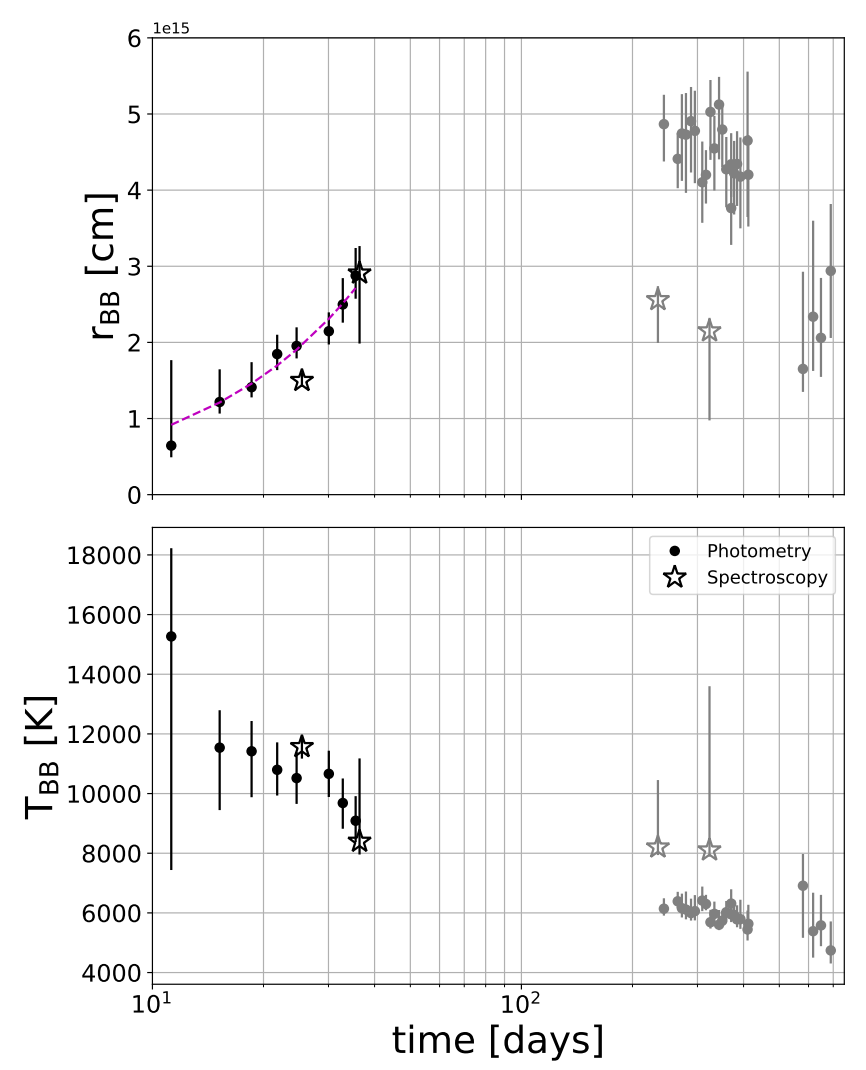

FIG. 6.- The evolution in time of: (1) the radius (top panel), (2) the temperature (lower panel) of a blackbody with the same radiation as PTF 12glz. The points were obtained by fitting a blackbody spectrum to the observed photometry, after interpolating the various data sets to obtain data coverage of coinciding epochs. The errors were obtained with Monte Carlo Markov chain simulations. The stars indicate the values derived by fitting a blackbody to the spectroscopic data. The dashed line in the top panel shows the best linear fit to the rising radius phase: a linear function with a slope of $\approx 8000 \mathrm{~km} \mathrm{~s}^{-1}$. At late times, the blackbody model for the spectral energy distribution may not be valid anymore (see e.g. right panel in figure 3): these points are shown in grey to emphasise that they are less reliable and should be taken cautiously.

ure 77. The evolution of $L$ should be taken cautiously, since at late time, a blackbody model for the spectral energy distribution may not be valid anymore, and so the

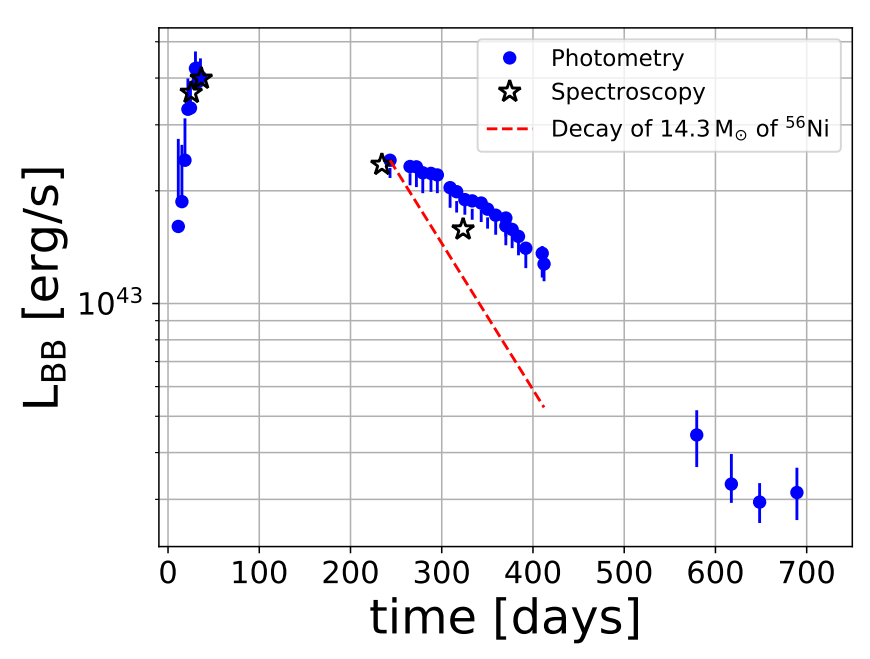

FIG. 7.- The evolution in time of the bolometric luminosity of a blackbody with the same radiation as PTF 12glz. The stars indicate the fits to the spectroscopic data. The dashed line shows the variation in luminosity caused by $14.3 \mathrm{M} \odot$ of ${ }^{56} \mathrm{Ni}$ radioactively decaying to ${ }^{56} \mathrm{Co}$ and then to ${ }^{56} \mathrm{Fe} . L_{B B}$ decays $\approx 2$ times slower, which suggests that radioactive decay is not sufficient to explain the decay of the light curve and that interaction or other sources of radiation play a role.

temperatures and radii used to calculate $L$ are less reliable.Although the late spectra analyzed in $\S 3.1$ suggest that the ejecta may have emerged through the CSM at late times, the slow decline of $L_{B B}$ hints at the fact that interaction may still play an important role in the radiation budget (this may happen through radiation from the reverse shock, or through processed radiation through the edge of the wind, or if the ejecta has only partially emerged through the CSM and is still interacting with it in some places).

\subsection{Mass of the CSM}

A very crude estimation of the swept-up CSM mass can be obtained by assuming that the ejecta mass is comparable to the mass of the CSM and that the bolometric luminosity $L$ is accounted for by the conversion of the kinetic energy of the ejecta into radiation:

$$
\int_{t} L(t) d t \lesssim \frac{1}{2} M_{\mathrm{CSM}} v_{e}^{2}
$$


where $M_{\mathrm{CSM}}$ is the mass of the CSM and $v_{e}$ is the velocity of the ejecta. To an order of magnitude, this estimate will not be very different than estimates based on more realistic treatment of the hydrodynamics (e.g., Ofek et al. 2014c). We use the width $F W H M \approx 8000 \mathrm{~km} \mathrm{~s}^{-1}$ of the broad $\mathrm{H} \alpha$ component at late time (see section 3.1) as an approximation of the ejecta velocity $v_{e}$. From the measured bolometric luminosity shown in Figure 7 $\int_{t} L(t) d t \approx 10^{51} \mathrm{erg}$. Substituting this value in Equation 2 gives $M_{\mathrm{CSM}} \gtrsim 2 M_{\odot}$.

In interacting SNe, the optically thick CSM masks the explosion, which may leave an ambiguity about the underlying explosion type. In particular, type Ia SNe and core-collapse SNe exploding inside a thick CSM would result in similar observational signatures. The spectra would look similar at early time (as long as the explosion is masked by the CSM) and at very late times, when $\mathrm{Ni}$ has decayed and there is no more energy to illuminate the ejecta and create absorption lines in the spectrum. Here, the high value of the bolometric luminosity excludes the possibility that PTF $12 \mathrm{glz}$ is a masked SN Ia.

Another order of magnitude estimate of the mass can be obtained by assuming a wind density profile, $\rho_{\mathrm{CSM}}(r)=K r^{-2}$ and using the photon diffusion timescale (e.g., Ofek et al. 2010),

$$
t_{d} \sim \frac{\kappa K}{c},
$$

where $\kappa$ is the CSM opacity. We assume $t_{d} \sim t_{c}$, where $t_{c}$ is the characteristic rise time of the bolometric light curve. We estimate $t_{c}$ by fitting the rising part of the bolometric light curve with the exponential function defined in Equation 1 and assuming $\kappa \approx 0.34 \mathrm{~cm}^{2} \mathrm{~g}^{-1}$. We obtain $t_{c} \sim 20$ day, and $K \approx 1.5 \times 10^{17} \mathrm{~g} \mathrm{~cm}^{-1}$, which corresponds to high values of the density $\rho_{\mathrm{CSM}} \gtrsim$ $10^{-14} \mathrm{~g} \mathrm{~cm}^{-3}$ (or a particle density of $n \sim 10^{10} \mathrm{~cm}^{-3}$, assuming a mean number of nucleons per particle $\left\langle\mu_{p}\right\rangle=$ $0.6)$ at the radii shown in Figure 6.

Measuring $K$ allows us to estimate the mass of the CSM swept up by the ejecta, $M_{\mathrm{CSM}}(r) \sim 4 \pi K r$ (assuming that the CSM extends on much higher scales than the stellar radius). Using the highest early-time radius $r_{B B}$, shown in Figure 6, as a lower limit of the maximum size of the shell of CSM surrounding the explosion, gives $M_{\mathrm{CSM}} \gtrsim 3 \mathrm{M}_{\odot}$, in good agreement with the estimates obtained with Equation 2

For a wind density profile, $K=\dot{M} /\left(4 \pi v_{w}\right)$, where $v_{w}$ is the velocity of the CSM. Therefore, measuring $K$ also gives us an estimation of the mass-loss rate. Using the width of the narrow $\mathrm{H} \alpha$ component during rise time, $F W H M \approx 200 \mathrm{~km} \mathrm{~s}^{-1}$, as a proxi of $v_{w}$ (see Table 4), we obtain a large mass-loss rate $\dot{M} \sim 0.6 M_{\odot} \mathrm{yr}^{-1}$, higher than the mass-loss rates observed in Taddia et al. (2013) and Kiewe et al. (2012) and comparable to the mass-loss rate of e.g., iPTF13z (Nyholm et al. 2017). Combining these estimates suggests that the CSM mass was ejected on a timescale of 1 to 10 years prior to the SN explosion.

We wish to emphasize that we made several simplifying assumptions in this section. In particular (1) we assumed a spherical symmetry of the CSM, (2) we assumed a wind profile of the CSM and (3) we assumed that the kinetic energy of the ejecta converts efficiently into radiation. Therefore, the numbers above have to be considered as order of magnitude estimates.

\subsection{Dust formation?}

At late times, the broad component of the $\mathrm{H} \alpha$ Balmer line is blueshifted by $\approx 1000 \mathrm{kms}^{-1}$ relative to the galaxy rest frame. The intermediate component is also blueshifted, but by a velocity of $\approx 250 \mathrm{~km} \mathrm{~s}^{-1}$ which is consistent with a typical stellar velocity within the galaxy (see Table 4 and Figure 5). Several explanations have been proposed to explain the blueshift of emission line profiles in interacting SN. One possible explanation is the formation of dust which increasingly blocks the receding parts of the ejecta (e.g., as proposed in the case of the Type IIn SN 2010 jl, Smith et al. 2012, Gall et al. 2014). In our case, the blueshift of the broad component does not grow with time (see Table 4), meaning that if there is dust, it may have formed before the epoch of the first nebular spectrum. In our case, testing the wavelength dependency of the blueshift - a blueshift caused by dust would be stronger at bluer wavelengths - is tricky because the blended iron emission lines mask the structure of the $H \beta$ line profile. We tried to apply several filters on this area, to separate the possible broad Balmer components from Fe II blend structures, but the results remained inconclusive.

Other explanations have been proposed for the blueshift of emission lines. Fransson et al. (2014), for example, attributed the blueshift of emission lines to radiative acceleration of the preshock gas by the SN radiation, whereas Smith et al. (2012) proposed a geometric explanation.

\section{RADIATIVE DIFFUSION THROUGH A SLAB}

The radiation from a SN exploding into a spherically symmetric CSM has been studied analytically, under simplifying assumptions (e.g., Chevalier 1982, Balberg \& Loeb 2011; Ginzburg \& Balberg 2014) and numerically (e.g., Falk \& Arnett 1977; Moriya \& Maeda 2014). The case of an aspherical CSM has been explored to a lesser extent (van Marle et al. 2010, McDowell et al. 2018). Exploring the expected effect of deviation from spherically symmetric CSM on the observables is all the more important since aspherical clouds of CSM around massejecting stars seem to be common, e.g., in stars like $\eta$ Carinae (Davidson \& Humphreys 1997) that have been proposed as SNe IIn progenitors (e.g., Gal-Yam et al. 2007; Gal-Yam \& Leonard 2009). In this section, we attempt to determine whether a non-spherical geometry of the CSM around a SN can explain the growing radius and decreasing temperature observed in $\S 3.2$. Solving for the exact shape of the CSM from a few observables in an illconditionned problem. Therefore, here our goal is merely to verify that a non-spherical geometry can explain the evolution of the observables. Given this goal, we consider a simple aspherical structure: a three-dimensional slab, infinite in two dimensions and perpendicular to the line of sight.

\subsection{Model and assumptions}

We have written a three dimensional computer program in python, SLAB-Diffusion, available on-line ${ }^{21}$ in

\footnotetext{
21 https://github.com/maayane/SLAB-Diffusion
} 


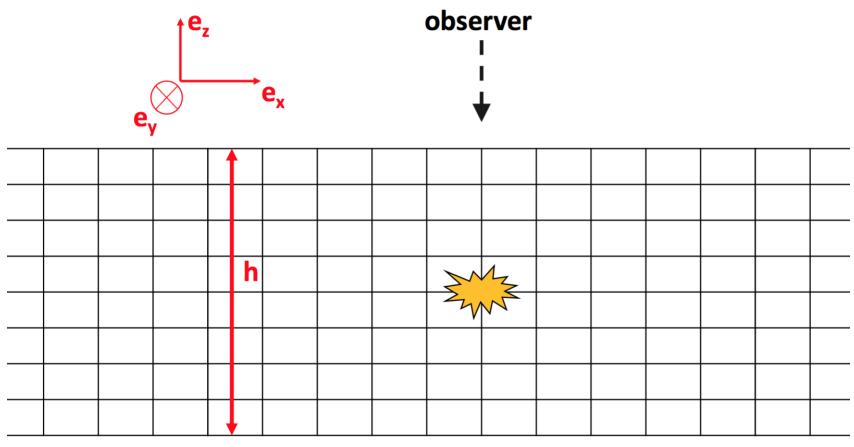

FIG. 8. - Sketch of the grid used to simulate radiative diffusion through a slab. The dimensions of the slab along the $e_{x}$ and $e_{y}$ coordinates are much larger than $h$, the dimension of the slab parallel to the line of sight. The initial energy of the explosion is deposited at $z=0$, at a distance $h / 2$ from the edge of the slab.

order to calculate the propagation of photons through a slab and simulate the main observables. The simple geometry we consider is illustrated in a cartoon in Figure 8. Following a similar approach as in Ginzburg \& Balberg (2014), we replaced the hydrodynamical description of the SN explosion by a stationary model of the shock breakout. This is equivalent to neglecting the expansion of the gas due to the explosion and modelling the interaction between the shock and the CSM as an instantaneous deposition of energy in the slab.

We assumed that the problem can be treated accurately within the diffusion approximation (i.e. assuming that $\tau \approx 1$ occurs close to the surface). Hence, the energy density $u$ within the slab is described by the diffusion equation:

$$
\frac{\partial u}{\partial t}=\nabla \cdot(D \vec{\nabla} u)
$$

where $D=c /(3 \kappa \rho)$ is the diffusion coefficient. Here we explored three density profiles: a constant density profile, $\rho$, and two functions of the $\vec{e}_{z}$ coordinate: $\rho \propto|z|^{-1}$ and $\rho \propto z^{-2}$, where the origin of the $z$-axis is at the center of the slab. We leave the treatment of the angular dependency of $D$ to further extensions of this work.

At the boundaries, the energy escapes from the slab and the flux $\vec{f}$ is linked to the density of energy through

$$
\|\vec{f}\|=\alpha c u,
$$

where $\alpha \approx 1 / 4$ (the case $\alpha=1 / 4$ corresponds to isotropic radiation). We discretized Equation 4 in a cartesian, three-dimensional grid (illustrated in a cartoon in Figure 8p, using an explicit forward Euler scheme with $\Delta t D /(\Delta d)^{2}<0.1$ where $\Delta d$ is defined for each coordinate and is the size of the mesh in each direction $\overrightarrow{e_{x}}$, $\overrightarrow{e_{y}}$ and $\overrightarrow{e_{z}}$. We assumed that $D$ does not depend on the wavelength of the photons (i.e. we made the so called grey approximation). We solved a dimensionless version of Equation 4 .

$$
\frac{\partial u}{\partial t^{\prime}}=\nabla \cdot\left(D^{\prime}(z) \vec{\nabla} u\right),
$$

where $t^{\prime}=t D(h) / h^{2}$ and $D^{\prime}(z)=D(z) / D(h)$. In this case the boundary conditions are

$$
\|\vec{f}\|=\alpha v u \quad, v=c / v_{d},
$$

where $v_{d}=D(h) / h$. A slab with a width $h=10^{16} \mathrm{~cm}$, an opacity $\kappa=0.34 \mathrm{~cm}^{2} \mathrm{~g}^{-1}$ and a constant mass density $\rho=1 \times 10^{-16} \mathrm{~g} \mathrm{~cm}^{-3}$ (corresponding e.g., to $3 M_{\odot}$ of CSM in a slab with $L_{x}=L_{y}=8 h$ ), corresponds to the unitless velocity $v \approx 1$ and a diffusion time $t_{d}=$ $h^{2} 3 \kappa \rho / c=94$ days.

In order to minimize the effect of the finite size of the grid along $\overrightarrow{e_{x}}$ and $\overrightarrow{e_{y}}$, we took several precautions. We chose the dimensions of the grid along the $\overrightarrow{e_{x}}$ and $\overrightarrow{e_{y}}$ directions so that $L_{x}=L_{y}$ and $L_{x} \gg h$. We checked that $L_{x}$ is large enough compared to $h$ so that a change in $L_{x}$ does not affect the results. Equation 7 only describes the boundary conditions along the $e_{\vec{z}}$ direction. We checked that we can apply reflective boundary conditions (i.e. $\vec{f}=\overrightarrow{0}$ at the boundaries) or absorbing boundary conditions (i.e. $u=0$ at the boundary) along the $\overrightarrow{e_{x}}$ and $\overrightarrow{e_{y}}$ directions, without affecting the results. We also checked for convergence of the code with respect to the time steps.

\subsection{Results}

As photons diffuse in the slab, they reach the $z=h / 2$ surface visible to the observer (see Figure 8). In Figure 9 we show the evolution of the total flux of energy escaping from the surface of a slab with $v=1$, in response to an instantaneous deposition of energy at $t=0$. We use the full-width at half maximum (FWHM) of the energy density $u$ at the surface of the slab as a proxy of the radius seen by the observer (below we present another proxy of $\left.r_{B B}\right)$. In Figure 10, we show that the FWHM grows in time for all three checked density profiles $\rho=$ Const., $\rho \propto|z|^{-1}$ and $\rho \propto z^{-2}$. We checked that varying the parameter $v$ does not change this qualitative result.

We would like to check whether our model can reproduce the decrease of the blackbody temperature $T_{B B}$ observed in Figure 6, in addition to producing a growing radius. Here again, given the simplicity of our model geometry, we are interested in the evolution of $T_{B B}$ rather than trying to fit its actual values. By modeling each cell of the $z=h / 2$ surface as a blackbody with temperature $T \propto u(x, y)^{1 / 4}$ and summing up all the cell spectra, we can compute the overall spectrum of the surface. The resultant spectrum is well represented by a blackbody spectrum, which allows us to deduce the blackbody temperature of the surface. This strategy also provides an additional way to recover the growing radius, by using:

$$
L=\int_{S} f d s=\sigma T_{B B}^{4} 4 \pi r_{B B}^{2} .
$$

In Figure 11, we show the evolution of the temperature $T_{B B}$ and radius $r_{B B}$, assuming $h=1 \times 10^{15} \mathrm{~cm}$, a constant mass density $\rho=3 \times 10^{-14} \mathrm{~g} \mathrm{~cm}^{-3}$ and an input energy of $10^{51} \mathrm{erg}$. Using this energy (calculated in Equation 2 as the energy initially deposited in the slab, is equivalent to assuming that all the energy radiated by the SN explosion is released by the time it starts diffusing out through the CSM. This is only correct within the simplistic assumption that the characteristic timescale for diffusion is much larger than the characteristic timescale for interaction between the ejecta and the CSM. Figure 11 shows that the aspherical geometry of the slab allows to recover the increase of the radius $r_{B B}$ and the decrease of the temperature $T_{B B}$ observed in Figure6. 


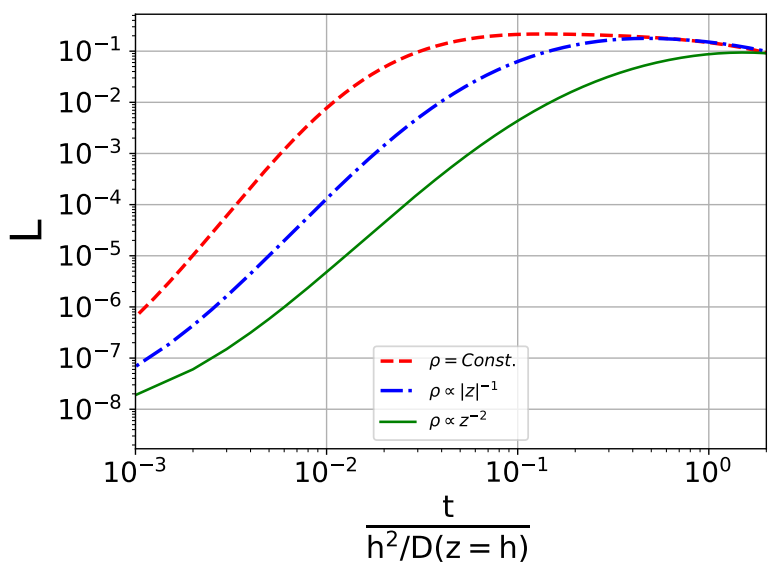

FIG. 9.- Luminosity $L=\int_{S} f d s$ released at the surface of a slab with $v=1$, normalized so that the initial energy in the slab is 1 . The luminosity is shown for a slab with a constant density profile (dashed line), a density profile $\rho \propto|z|^{-1}$ (dotted line) and a wind density profile $\rho \propto z^{-2}$ (continuous line).

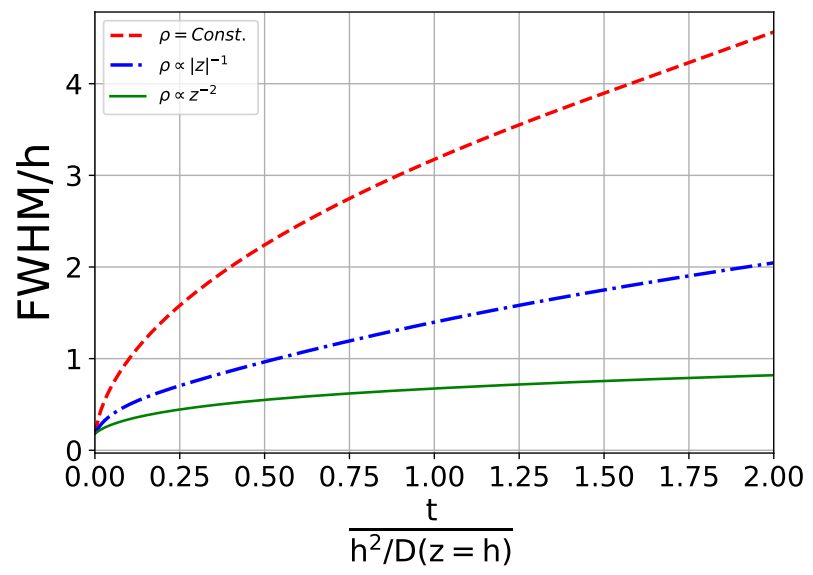

FIG. 10.- Full width at half-maximum (FWHM) of the density of energy $u$ at the surface of a slab with $v=1$. The FWHM is used as a proxy of the black body radius measured by the observer. The FWHM is shown for a slab with a constant density profile (dashed line), a density profile $\rho \propto|z|^{-1}$ (dotted line) and a wind density profile $\rho \propto z^{-2}$ (continuous line).

\section{CONCLUSIONS}

We presented the observations of the supernova PTF $12 \mathrm{glz}$ by the GALEX space telescope and groundbased PTF. Radioactive decay is not sufficient to explain the decay of the light curve of PTF 12glz and therefore other physical mechanisms must be involved. One possible - yet difficult to verify - scenario is that an internal engine powers the light curve. Another possible scenario - the standard explanation invoked in the case of Type IIn $\mathrm{SNe}$, is that the light curve is powered by interaction between the ejecta and the CSM surrounding the SN.

In the case of PTF 12glz, the spectroscopic analysis is consistent with the following picture: at early times (two first spectra) both the ejecta and the shock are initially masked by a thick, slowly moving, photoionized CSM. At later times (two last spectra), the ejecta have emerged through - at least some of - the optically thick layers and have reached CSM layers that are optically thin enough to expose the ejecta. CSM interaction may still play a

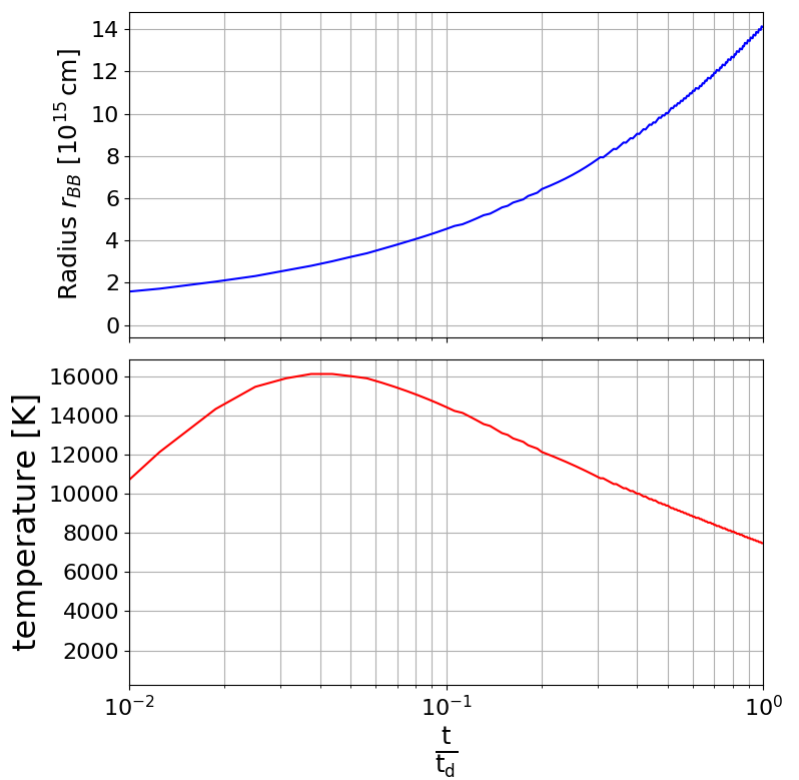

FIG. 11. - The evolution in time of: (1) the blackbody radius $r_{B B}$ (top panel) and (2) the blackbody temperature (lower panel) at the surface of a slab with constant density. The diffusion equation was solved with an input energy $E_{i}=10^{51} \mathrm{erg}$ deposited in a slab of width $h=10^{16} \mathrm{~cm}$ and constant mass density $\rho=1 \times 10^{-16} \mathrm{~g} \mathrm{~cm}^{-3}$, corresponding to $t_{d}=h^{2} 3 \kappa \rho / c=94$ days. The spectrum of the $z=h / 2$ surface was deduced by summing the blackbody spectra of all the cells of the surface and $T_{B B}$ was then deduced by fitting a blackbody to the resultant spectrum. The blackbody radius $r_{B B}$ was deduced through the relation $r_{B B}=\sqrt{L /\left(4 \pi \sigma T^{4}\right)}$. The aspherical geometry of the slab allows to recover the increase of $r_{B B}$ and the decrease in $T_{B B}$.

role at late times, e.g., by heating the ejecta from the inside, and contributes to slowing the light curve decay.

The evolution of $r_{B B}$ - the radius of the deepest transparent emitting layer - seems to contradict this picture. At early times, i.e., at the very time when the opaque CSM seemingly obstructs our view of any growing structure, $r_{B B}$ grows by an order of magnitude, at a speed of $\sim 8000 \mathrm{~km} \mathrm{~s}^{-1}$. In addition to being inconsistent with the spectroscopic analysis, this is also in contradiction to our knowledge - with all previous observations of either a constant or stalling blackbody radius in SNe IIn (as detailed in $\S 3.2$ ).

If the bulk of the radiation from PTF 12glz does come from interaction, the explanation for the growing blackbody radius may be geometrical. The question then is whether any peculiar structure of the CSM around the progenitor can reproduce the observations. In this work, we considered a simple aspherical structure of CSM: a slab. We modeled the radiation from an explosion embedded in a slab of CSM by numerically solving the radiative diffusion equation in a slab with different density profiles: $\rho=$ Const., $\rho \propto|z|^{-1}$ and a wind density profile $\rho \propto z^{-2}$. Although this model is simplistic, it allows recovery of the peculiar growth of the blackbody radius $r_{B B}$ observed in the case of PTF 12glz, as well as the decrease of its blackbody temperature $T_{B B}$. This configuration is not a unique geometrical solution and additional observations, e.g., of the polarization around PTF 12glz would have been necessary to make it less speculative.

As new wide-field transient surveys such as the Zwicky 
Transient Facility (e.g., Bellm et al. 2015; Laher et al. 2017) are deployed, many more interacting SNe will be observed and quickly followed up with multiple-band observations. These may also be the brightest sources for the ULTRASATUV satellite mission (Sagiv et al. 2014). Some of these interacting SNe may exhibit the same peculiarities as PTF 12glz. The methodology proposed in this paper offers a framework to analyze them. It could be elaborated upon, to model more complex aspherical geometries, e.g., $\eta$ Carinae-like shapes of the CSM, and give more quantitative predictions of the observables.

M.T.S. thanks Jonathan Morag, Adam Rubin, Yi Yang, Doron Kushnir, Anders Nyholm and Chalsea Harris for useful discussions. M.T.S. acknowledges support by a grant from IMOS/ISA, the Ilan Ramon fellowship from the Israel Ministry of Science and Technology and the Benoziyo center for Astrophysics at the Weizmann Institute of Science.

E.O.O is grateful for the support by grants from the Israel Science Foundation, Minerva, Israeli Ministry of Science, the US-Israel Binational Science Foundation, the Weizmann Institute and the I-CORE Program of the Planning and Budgeting Committee and the Israel Science Foundation.

A.G.-Y. is supported by the EU via ERC grant No. 725161, the Quantum Universe I-Core program, the ISF, the BSF Transformative program, IMOS via ISA and by a Kimmel award.

\section{REFERENCES}

Aretxaga, I., Benetti, S., Terlevich, R. J., et al. 1999, MNRAS, 309,343

Balberg, S., \& Loeb, A. 2011, MNRAS, 414, 1715

Bellm, E. C., Kulkarni, S. R., \& ZTF Collaboration. 2015, in American Astronomical Society Meeting Abstracts, Vol. 225, American Astronomical Society Meeting Abstracts \#225, 328.04

Bilinski, C., Smith, N., Williams, G. G., et al. 2017, arXiv: 1712.03370

Boles, T., Nakano, S., \& Itagaki, K. 2005, Central Bureau Electronic Telegrams, 275

Cardelli, J. A., Clayton, G. C., \& Mathis, J. S. 1989, ApJ, 345, 245

Cenko, S. B., Fox, D. B., Moon, D.-S., et al. 2006, PASP, 118, 1396

Chevalier, R. A. 1982, ApJ, 258, 790

- 2012, ApJ, 752, L2

Chevalier, R. A., \& Fransson, C. 1994, ApJ, 420, 268

Chevalier, R. A., \& Fransson, C. 2003, in Lecture Notes in Physics, Berlin Springer Verlag, Vol. 598, Supernovae and Gamma-Ray Bursters, ed. K. Weiler, 171-194

Chevalier, R. A., \& Irwin, C. M. 2011, The Astrophysical Journal Letters, 729, L6

Chevalier, R. A., \& Irwin, C. M. 2012, ApJ, 747, L17

Chugai, N. N. 2001, MNRAS, 326, 1448

Chugai, N. N., \& Danziger, I. J. 1994, MNRAS, 268, 173

Chugai, N. N., Blinnikov, S. I., Cumming, R. J., et al. 2004, MNRAS, 352, 1213

Danziger, I. J., \& Kjaer, K., eds. 1991, European Southern Observatory Conference and Workshop Proceedings, Vol. 37, Supernova 1987A and other supernovae

Davidson, K., \& Humphreys, R. M. 1997, ARA\&A, 35, 1

Davidson, K., \& Humphreys, R. M., eds. 2012, Astrophysics and Space Science Library, Vol. 384, Eta Carinae and the Supernova Impostors

Dilday, B., Howell, D. A., Cenko, S. B., et al. 2012, Science, 337, 942

Elias-Rosa, N., Pastorello, A., Benetti, S., et al. 2016, MNRAS, 463,3894

Fabian, A. C., \& Terlevich, R. 1996, MNRAS, 280, L5

Falk, S. W., \& Arnett, W. D. 1977, ApJS, 33, 515

Fassia, A., Meikle, W. P. S., Vacca, W. D., et al. 2000, MNRAS, 318,1093

Fassia, A., Meikle, W. P. S., Chugai, N., et al. 2001, MNRAS, 325, 907

Filippenko, A. V. 1997, ARA\&A, 35, 309

Firth, R. E., Sullivan, M., Gal-Yam, A., et al. 2015, MNRAS, 446,3895

Foley, R. J., Smith, N., Ganeshalingam, M., et al. 2007, ApJ, 657 L105

Foreman-Mackey, D., Hogg, D. W., Lang, D., \& Goodman, J. 2013, Publications of the ASP, 125, 306

Fransson, C., Ergon, M., Challis, P. J., et al. 2014, ApJ, 797, 118

Fraser, M., Magee, M., Kotak, R., et al. 2013, ApJ, 779, L8

Fremling, C., Sollerman, J., Taddia, F., et al. 2016, A\&A, 593, A68

Gal-Yam, A. 2016, ArXiv e-prints, arXiv:1611.09353

Gal-Yam, A., \& Leonard, D. C. 2009, Nature, 458, 865

Gal-Yam, A., Leonard, D. C., Fox, D. B., et al. 2007, ApJ, 656 372

Gal-Yam, A., Arcavi, I., Ofek, E. O., et al. 2014, Nature, 509, 471

Gall, C., Hjorth, J., Watson, D., et al. 2014, Nature, 511, 326

Ganot, N., Gal-Yam, A., Ofek, E. O., et al. 2016, ApJ, 820, 57
Ginzburg, S., \& Balberg, S. 2014, ApJ, 780, 18

Graham, M. L., Harris, C. E., Fox, O. D., et al. 2017, ApJ, 843, 102

Hoffman, J. L., Leonard, D. C., Chornock, R., et al. 2008, ApJ, 688,1186

Hogg, D. W., Bovy, J., \& Lang, D. 2010, arXiv:1008.4686

Katz, B., Sapir, N., \& Waxman, E. 2011, arXiv:1106.1898

Khazov, D., Yaron, O., Gal-Yam, A., et al. 2016, ApJ, 818, 3

Kiewe, M., Gal-Yam, A., Arcavi, I., et al. 2012, ApJ, 744, 10

Kuncarayakti, H., Maeda, K., Ashall, C. J., et al. 2018, ApJ, 854, L14

Laher, R. R., Surace, J., Grillmair, C. J., et al. 2014, PASP, 126, 674

Laher, R. R., Masci, F. J., Groom, S., et al. 2017, ArXiv e-prints, arXiv: 1708.01584

Law, N. M., Kulkarni, S. R., Dekany, R. G., et al. 2009, PASP, 121,1395

Leonard, D. C., Filippenko, A. V., Barth, A. J., \& Matheson, T. 2000, ApJ, 536, 239

Li, W.-D., Li, C., Filippenko, A. V., \& Moran, E. C. 1998, IAU Circ., 6829

McDowell, A. T., Duffell, P. C., \& Kasen, D. 2018, arXiv: 1802.05152

Moriya, T. J., \& Maeda, K. 2014, ApJ, 790, L16

Murase, K., Thompson, T. A., Lacki, B. C., \& Beacom, J. F. 2011, Phys. Rev. D, 84, 043003

Murase, K., Thompson, T. A., \& Ofek, E. O. 2014, MNRAS, 440, 2528

Nakar, E., \& Sari, R. 2010, ApJ, 725, 904

Nyholm, A., Sollerman, J., Taddia, F., et al. 2017, A\&A, 605, A6

Ofek, E. O. 2014, MATLAB package for astronomy and astrophysics, Astrophysics Source Code Library, ascl:1407.005

Ofek, E. O., Cameron, P. B., Kasliwal, M. M., et al. 2007, ApJ, 659, L13

Ofek, E. O., Rabinak, I., Neill, J. D., et al. 2010, The Astrophysical Journal, 724, 1396

Ofek, E. O., Laher, R., Law, N., et al. 2012, PASP, 124, 62

Ofek, E. O., Sullivan, M., Cenko, S. B., et al. 2013a, Nature, 494, 65

Ofek, E. O., Fox, D., Cenko, S. B., et al. 2013b, ApJ, 763, 42

Ofek, E. O., Arcavi, I., Tal, D., et al. 2014a, ApJ, 788, 154

Ofek, E. O., Sullivan, M., Shaviv, N. J., et al. 2014b, ApJ, 789, 104

Ofek, E. O., Zoglauer, A., Boggs, S. E., et al. 2014c, ApJ, 781, 42

Ofek, E. O., Cenko, S. B., Shaviv, N. J., et al. 2016, ApJ, 824, 6

Oke, J. B., \& Gunn, J. E. 1982, PASP, 94, 586

Oke, J. B., Cohen, J. G., Carr, M., et al. 1994, in Proc. SPIE, Vol. 2198, Instrumentation in Astronomy VIII, ed. D. L. Crawford \& E. R. Craine, 178-184

Pastorello, A., Smartt, S. J., Mattila, S., et al. 2007, Nature, 447, 829

Patat, F., Taubenberger, S., Benetti, S., Pastorello, A., \& Harutyunyan, A. 2011, A\&A, 527, L6

Quataert, E., \& Shiode, J. 2012, MNRAS, 423, L92

Rabinak, I., \& Waxman, E. 2011, ApJ, 728, 63

Rahmer, G., Smith, R., Velur, V., et al. 2008, in Proc. SPIE, Vol. 7014, Ground-based and Airborne Instrumentation for Astronomy II, 70144Y

Rau, A., Kulkarni, S. R., Law, N. M., et al. 2009, PASP, 121, 1334

Reilly, E., Maund, J. R., Baade, D., et al. 2017, MNRAS, 470 1491

Rest, A. 2009, IAU Circ., 9092

Rest, A., Foley, R. J., Gezari, S., et al. 2011, ApJ, 729, 88 
Sagiv, I., Gal-Yam, A., Ofek, E. O., et al. 2014, AJ, 147, 79 Schlafly, E. F., \& Finkbeiner, D. P. 2011, ApJ, 737, 103

Schlegel, E. M. 1990, MNRAS, 244, 269

Schlegel, E. M., \& Petre, R. 2006, ApJ, 646, 378

Silverman, J., Nugent, P. E., Gal-Yam, A., et al. 2013, in American Astronomical Society Meeting Abstracts, Vol. 221, American Astronomical Society Meeting Abstracts \#221, 253.06

Smith, N. 2014, ARA\&A, 52, 487

Smith, N., Chornock, R., Li, W., et al. 2008, ApJ, 686, 467

Smith, N., \& McCray, R. 2007, ApJ, 671, L17

Smith, N., Silverman, J. M., Filippenko, A. V., et al. 2012, AJ, 143,17

Smith, N., Silverman, J. M., Chornock, R., et al. 2009, ApJ, 695, 1334

Smith, N., Kilpatrick, C. D., Mauerhan, J. C., et al. 2017, MNRAS, 466, 3021

Soker, N., \& Kashi, A. 2016, MNRAS, 462, 217

Stathakis, R. A., \& Sadler, E. M. 1991, MNRAS, 250, 786

Stoll, R., Prieto, J. L., Stanek, K. Z., et al. 2011, ApJ, 730, 34

Stritzinger, M., Taddia, F., Fransson, C., et al. 2012, ApJ, 756, 173

Sullivan, M., Howell, D. A., Perrett, K., et al. 2006, AJ, 131, 960

Svirski, G., Nakar, E., \& Sari, R. 2012, ApJ, 759, 108
Taddia, F., Stritzinger, M. D., Sollerman, J., et al. 2013, A\&A, 555, A10

Thöne, C. C., de Ugarte Postigo, A., Leloudas, G., et al. 2017, A\&A, 599, A129

Thrasher, P., Li, W., \& Filippenko, A. V. 2008, Central Bureau Electronic Telegrams, 1507

Turatto, M., Cappellaro, E., Danziger, I. J., et al. 1993, MNRAS, 262,128

van Dyk, S. D., Weiler, K. W., Sramek, R. A., \& Panagia, N. 1993, ApJ, 419, L69

van Marle, A. J., Smith, N., Owocki, S. P., \& van Veelen, B. 2010, MNRAS, 407, 2305

Wang, L., \& Wheeler, J. C. 2008, ARA\&A, 46, 433

Weaver, T. A. 1976, ApJS, 32, 233

Williams, C. L., Panagia, N., Van Dyk, S. D., et al. 2002, ApJ, 581,396

Woosley, S. E., Blinnikov, S., \& Heger, A. 2007, Nature, 450, 390

Yaron, O., \& Gal-Yam, A. 2012, PASP, 124, 668

Yaron, O., Perley, D. A., Gal-Yam, A., et al. 2017, Nature Physics, 13, 510

York, D. G., Adelman, J., Anderson, Jr., J. E., et al. 2000, AJ, 120,1579 